\title{
The Chemistry of Environmental Interfaces: A Joined X-ray Spectromicroscopy and NanoSIMS Approach
}

M Keiluweit*, PS Nico**, LH Zeglin*, J Pett-Ridge***, P Weber***, DD Myrold*, and M Kleber*

* Department of Crop and Soil Science, Soils Division, Oregon State University, Corvallis, OR

** Lawrence Berkeley National Laboratory, Earth Sciences Division, Berkeley, CA

*** Lawrence Livermore National Laboratory, Chemical Sciences Division, Livermore, CA

Plant roots, microbial bodies and derived compounds are likely the main source of carbon sequestered and stabilized in soil [1]. Most root carbon is decomposed to $\mathrm{CO}_{2}$, and the remainder typically undergoes multiple microbial transformations before it is stabilized. The characteristics of the resulting plant and microbially derived organic compounds (lipids, proteins, polysaccharides, lignins, amino acids) are likely to interact differently with soils mineral phases in soils, which will determine how much carbon remains in soil based on the reactivity of their component molecular groups (carboxylic acids, amines, aromatic rings) [2]. Advancing our understanding of soil organic matter dynamics requires disentangling the complex interactions between soil mineral surfaces and decomposed organic compounds, and soil microbial activity/biomass.

To better understand the interactions between soil organic matter, microbiota and mineral surfaces, we are marrying two cutting-edge technologies: high-resolution imaging spectroscopy (STXM/NEXAFS) and high-resolution imaging mass spectrometry (NanoSIMS). This unique combination allows us to map carbon and nitrogen distribution, image associations of organics with specific mineral types, and trace organic matter of differing origins into the soil matrix. However, it requires that a sample be mounted and examined on two different analytical instruments. In view of the extremely smattscales involved, this poses a significant technical challenge. Therefore, our technological goal is to develop novel preparation techniques for complex environmental samples and new ways to navigate and re-locate analysis sites using these two complementary instruments.

Our scientific goal is to provide data to improve modeling and management of soil resources. A mechanistic understanding of interactions at the interface between organic materials, microbiota and minerals in soils is crucial to the maintenance of sustainable organic matter levels in soils, especially in systems stressed by both climate change and intensive agriculture.

Here we demonstrate the technically feasibility of conducting these complementary analyses on the same environmental sample - model microstructures consisting of associations between soil minerals and ${ }^{13} \mathrm{C}$ - and ${ }^{15} \mathrm{~N}$-labeled microbial cell residues. The objective of this initial experiment is to establish a routine that affords the opportunity to study carbon and nitrogen dynamics within individual soil microstructures.

Our technical approach is to:

(i) Identify microstructures of particular interest and their surface topography via scanning electron microscopy (SEM). 
(ii) Analyze the surface chemistry (spatial distribution and speciation of $\mathrm{C}$ and $\mathrm{N}$ ) of the same microstructure using a synchrotron-based scanning X-ray microscopy (STXM) combined with near-edge X-ray absorption fine structure (NEXAFS) spectroscopy.

(iii) Extract quantitative maps of surface-associated biopolymers (proteins, lipids, and polysaccharides) from STXM/NEXAFS image sequences measured at the Carbon K-edge by using singular value decomposition (SVD) to fit the spectrum at each pixel to a linear combination of reference spectra of the components.

(iv) Probe the distribution of ${ }^{13} \mathrm{C}$ - and ${ }^{15} \mathrm{~N}$-enriched cell components across the microstructure surface using NanoSIMS.

Our presentation will illustrate the successful investigation of identical soil microstructures on both the synchrotron-based X-ray spectromicroscopy (STXM/NEXAFS) at the Advance Light Source (ALS) beamline 5.3.2 and subsequently at the high-resolution imaging mass spectrometer (NanoSIMS) at Lawrence Livermore National Laboratory. We will show how this joint approach facilitates investigations of the chemistry of surfaces and interfaces in complex environmental samples. Finally, logistics and technical limitations of the combined SEMNEXAFS/STXM-NanoSIMS analysis on environmental samples will be discussed.

\section{References:}

[1] Rasse DP, Rumpel C, Dignac M (2005). Is soil carbon mostly root carbon? Mechanisms for a specific stabilisation. Plant and Soil 269: 341-356.

[2] Kuzyakov Y (2002). Review: Factors affecting rhizosphere priming effects. J. Plant Nutr. Soil Sci 165(4): 382-396. 